\title{
Urine proteome changes in rats subcutaneously inoculated with approximately ten tumor cells
}

\author{
Jing Wei ${ }^{1}$, Wenshu Meng ${ }^{1}$, Youhe Gao ${ }^{\text {Corresp. } 1}$ \\ ${ }^{1}$ Department of Biochemistry and Molecular Biology, Beijing Normal University, Gene Engineering and Biotechnology Beijing Key Laboratory, Beijing, \\ China \\ Corresponding Author: Youhe Gao \\ Email address: gaoyouhe@bnu.edu.cn
}

Background: Biomarkers are changes associated with the disease. Urine is not subject to homeostatic control and therefore accumulates very early changes, making it an ideal biomarker source. Usually, we have performed urinary biomarker studies involving at least thousands of tumor cells. However, no tumor starts from a thousand tumor cells. We therefore examined urine proteome changes in rats subcutaneously inoculated with approximately ten tumor cells.

Methods: Here, we serially diluted Walker-256 carcinosarcoma cells to a concentration of $10^{2} / \mathrm{mL}$ and subcutaneously inoculated $0.1 \mathrm{~mL}$ of these cells into nine rats. The Urine proteomes on days 0,13 and 21 were analyzed by liquid chromatography coupled with tandem mass spectrometry.

Results: Hierarchical clustering analysis showed that the urine proteome of each sample at three time points were clustered into three clusters, indicating the good consistency of these nine rats when inoculated with the same limited tumor cells. Differential proteins on days 13 and 21 were mainly associated with cell adhesion, autophagic cell death, changes in extracellular matrix organization, angiogenesis, and the pentose phosphate pathway. All of these enriched functional processes were reported to contribute to tumor progression and could not be enriched through random allocation analysis.

Conclusions: Our results indicated that 1 ) the urine proteome reflects changes associated with cancer even with only approximately ten tumor cells in the body and that 2) the urine proteome reflects pathophysiological changes in the body with extremely high sensitivity and provides potential for a very early screening process of clinical patients. 
1 Urine proteome changes in rats subcutaneously inoculated with approximately ten

3

4 Jing Wei ${ }^{1}$, Wenshu Meng ${ }^{1} \&$ Youhe Gao $^{1 *}$

$5 \quad{ }^{1}$ Department of Biochemistry and Molecular Biology, Beijing Normal University, Gene

6 Engineering Drug and Biotechnology Beijing Key Laboratory, Beijing, 100875, China

7 Corresponding author:

8 Youhe Gao

9 Email address: gaoyouhe@bnu.edu.cn

Tel.: +861058804382 


\section{Manuscript to be reviewed}

\section{Abstract}

40 Background: Biomarkers are changes associated with the disease. Urine is not subject to homeostatic control and therefore accumulates very early changes, making it an ideal biomarker source. Usually, we have performed urinary biomarker studies involving at least thousands of tumor cells. However, no tumor starts from a thousand tumor cells. We therefore examined urine proteome changes in rats subcutaneously inoculated with approximately ten tumor cells.

Methods: Here, we serially diluted Walker-256 carcinosarcoma cells to a concentration of $10^{2} / \mathrm{mL}$ and subcutaneously inoculated $0.1 \mathrm{~mL}$ of these cells into nine rats. The urine proteomes on days 0,13 and 21 were analyzed by liquid chromatography coupled with tandem mass spectrometry.

Results: Hierarchical clustering analysis showed that the urine proteome of each sample at three time points were clustered into three clusters, indicating the good consistency of these nine rats when inoculated with the same limited tumor cells. Differential proteins on days 13 and 21 were mainly associated with cell adhesion, autophagic cell death, changes in extracellular matrix organization, angiogenesis, and the pentose phosphate pathway. All of these enriched functional processes were reported to contribute to tumor progression and could not be enriched through random allocation analysis.

Conclusions: Our results indicated that 1) the urine proteome reflects changes associated with cancer even with only approximately ten tumor cells in the body and that 2) the urine proteome reflects pathophysiological changes in the body with extremely high sensitivity and provides the potential for a very early screening process of clinical patients.

\section{Introduction}

Urine is an ideal biomarker resource. Blood often remains stable because of homeostatic mechanisms. However, as the filtrate of blood, urine has no need to remain stable and thus tolerates a much higher degree of changes. Therefore, urine can accumulate all changes from the whole body and may provide the potential to detect early and small changes in the body (Gao 2013). Urine is easily affected by various physiological factors, such as sex, age and diet (Wu \& Gao 2015). In patients, the urine proteome is easily influenced by certain medications because of necessary therapeutic measures. Therefore, our laboratory proposed a strategy for urinary biomarker studies. First, we used animal models to find early biomarkers of related diseases. Then, we verified candidate biomarkers in clinical urine samples (Gao 2014). The use of animal models minimizes external influencing factors, such as diet, gender, age, medications and some environmental factors. In addition, using animal models will allow identification of the exact start of the disease, which is helpful in the early detection of cancer. Differential urinary proteins found in animal models are likely to be directly associated with related diseases. According to this strategy, our laboratory has applied different types of animal models, such as subcutaneous tumor-bearing model(Wu et al. 2017a), pulmonary

81 fibrosis model(Wu et al. 2017b), glioma model(Ni et al. 2018b), liver fibrosis model (Zhang Peer) reviewing PDF | (2019:05:37656:1:1:CHECK 31 Jul 2019) 


\section{Manuscript to be reviewed}

82 et al. 2018a), Alzheimer's disease model(Zhang et al. 2018b), chronic pancreatitis

83

84

85

86

87

88

89

90

91

92

93

94

95

96

97

98

99

100

101

102

103

104

105

106

107

108

109

110

111

112

113

114

115

116

117

118

119

120

121

122

123

model(Zhang et al. 2018c) and myocarditis model(Zhao et al. 2018), to search for early biomarkers before pathological changes and clinical manifestations.

Urine can reflect changes more sensitively than blood. It has been reported that even when interference is introduced into the blood with two anticoagulants, changes in the abundance of more proteins were consistently detected in urine samples than in plasma(Li et al. 2014). In addition, the urine proteome has been applied to detect tumors in various tumor-bearing animals. For example, i) in W256 subcutaneously tumor-bearing rats, a total of ten differential urinary proteins were identified before a tumor mass was palpable( $\mathrm{Wu}$ et al. 2017a); ii) in the intracerebral W256 tumor model, nine urinary proteins changed significantly before any obvious clinical manifestations or abnormal magnetic resonance imaging (MRI) signals (Zhang et al. 2019); iii) in the glioma rat model, a total of thirty differential proteins were identified before MRI ( $\mathrm{Ni}$ et al. 2018a); iv) a total of seven urinary proteins changed in both lung tumor-bearing mice and lung cancer patients, indicating their potential roles in the early detection of lung cancer (Zhang et al. 2015); v) in a urothelial carcinoma rat model, differential urinary proteins from upregulated biological processes might be seen as candidate biomarkers (Ferreira et al. 2015). All of these studies were performed involving thousands of tumor cells; however, no tumor starts from a thousand tumor cells. Since urine is a more sensitive biomarker resource than blood, we explored the sensitivity limit of urine. We determined whether the urine proteome changes if there are only a small number of tumor cells in the body.

In this study, we subcutaneously injected approximately ten Walker-256 carcinosarcoma cells into nine rats. Urine samples were collected on days 0,13 , and 21 . Urine proteins were analyzed by liquid chromatography-tandem mass spectrometry (LC-MS/MS). Differential proteins on days 13 and 21 were analyzed by functional enrichment analysis to find associations with tumor progression. This research aimed to determine whether the urine proteome could reflect changes associated with these ten tumor cells. The technical flowchart is presented in Figure 1.

\section{Materials and methods}

\section{Animal treatment}

Male Wistar rats $(n=12,150 \pm 20 \mathrm{~g})$ were purchased from Beijing Vital River Laboratory Animal Technology Co., Ltd. Animals were maintained with a standard laboratory diet under controlled indoor temperature $\left(21 \pm 2^{\circ} \mathrm{C}\right)$, humidity $(65-70 \%)$ and $12 \mathrm{~h} / 12 \mathrm{~h}$ light-dark cycle conditions. The experiment was approved by Peking Union Medical College (Approval ID: ACUC-A02-2014-008).

Walker-256 (W256) carcinosarcoma cells were purchased from the Cell Culture Center of the Chinese Academy of Medical Sciences (Beijing, China). W256 tumor cells were intraperitoneally inoculated into Wistar rats. The W256 ascites tumor cells were harvested from the peritoneal cavity after seven days. After two cell passages, the W256 ascites tumor cells were collected, centrifuged, and resuspended in $0.9 \%$ normal saline (NS). Then, W256 tumor cells were serially diluted to a concentration of $10^{2} / \mathrm{mL}$. The viability of W256 cells 
was evaluated by the Trypan blue exclusion test using a Neubauer chamber, and only $95 \%$ viable tumor cells were used for serially dilution.

The rats were randomly divided into the following two groups: rats subcutaneously inoculated with tumor cells $(n=9)$ and control rats $(n=3)$. In the experimental group, rats were inoculated with $10 \mathrm{~W} 256$ cells in $100 \mu \mathrm{L}$ of NS into the right flank of the rats. The control rats were subcutaneously inoculated with an equal volume of NS. All rats were anesthetized with sodium pentobarbital solution $(4 \mathrm{mg} / \mathrm{kg})$ before inoculation.

\section{Urine collection}

Before urine collection, all rats were accommodated in metabolic cages for 2-3 days. Urine samples were collected from rats subcutaneously inoculated with tumor cells $(n=9)$ on days 0,13 and 21. All rats were placed in metabolic cages individually for $12 \mathrm{~h}$ to collect urine without any treatment. After collection, urine samples were stored immediately at $-80^{\circ} \mathrm{C}$.

\section{Extraction and digestion of urinary proteins}

Urine samples $(n=27)$ were centrifuged at $12,000 \times \mathrm{g}$ for $30 \mathrm{~min}$ at $4^{\circ} \mathrm{C}$. Then, the supernatants were precipitated with three times the volume of ethanol at $-20^{\circ} \mathrm{C}$ overnight. The pellets were dissolved sufficiently in lysis buffer $(8 \mathrm{~mol} / \mathrm{L}$ urea, $2 \mathrm{~mol} / \mathrm{L}$ thiourea, 50 $\mathrm{mmol} / \mathrm{L}$ Tris, and $25 \mathrm{mmol} / \mathrm{L}$ DTT). After centrifugation at $4^{\circ} \mathrm{C}$ and $12,000 \times \mathrm{g}$ for $30 \mathrm{~min}$, the protein samples were measured by using the Bradford assay. A total of $100 \mu \mathrm{g}$ of each protein sample was digested with trypsin (Trypsin Gold, Mass Spec Grade, Promega, Fitchburg, Wisconsin, USA) by using filter-aided sample preparation (FASP) methods(Wisniewski et al. 2009). These digested peptides were desalted using Oasis HLB cartridges (Waters, Milford, MA) and then dried by vacuum evaporation (Thermo Fisher Scientific, Bremen, Germany).

\section{LC-MS/MS analysis}

Digested peptides $(n=27)$ were dissolved in $0.1 \%$ formic acid to a concentration of 0.5 $\mu \mathrm{g} / \mu \mathrm{L}$. For analysis, $1 \mu \mathrm{g}$ of peptide from each sample was loaded into a trap column $(75 \mu \mathrm{m}$ $\times 2 \mathrm{~cm}, 3 \mu \mathrm{m}, \mathrm{C} 18,100 \AA$ ) at a flow rate of $0.25 \mu \mathrm{L} / \mathrm{min}$ and then separated with a reversed-phase analytical column $(75 \mu \mathrm{m} \times 250 \mathrm{~mm}, 2 \mu \mathrm{m}, \mathrm{C} 18,100 \AA)$. Peptides were eluted with a gradient extending from $4 \%-35 \%$ buffer B $(0.1 \%$ formic acid in $80 \%$ acetonitrile) for $90 \mathrm{~min}$ and then analyzed with an Orbitrap Fusion Lumos Tribrid Mass Spectrometer (Thermo Fisher Scientific, Waltham, MA). The MS data were acquired using the following parameters: i) data-dependent MS/MS scans per full scan were auquired at the top-speed mode; ii) MS scans had a resolution of 120,000, and MS/MS scans had a resolution of 30,000 in Orbitrap; iii) HCD collision energy was set to $30 \%$; iv) dynamic exclusion was set to $30 \mathrm{~s}$; v) the charge-state screening was set to +2 to +7 ; and vi) the maximum injection time was $45 \mathrm{~ms}$. Each peptide sample was analyzed twice.

\section{Label-free quantification}

Raw data files $(n=54)$ were searched using Mascot software (version 2.5.1, Matrix Science, London, UK) against the Swiss-Prot rat database (released in February 2017, containing 7,992 PeerJ reviewing PDF | (2019:05:37656:1:1:CHECK 31 Jul 2019) 
167 sequences). The parent ion tolerance was set to $10 \mathrm{ppm}$, and the fragment ion mass tolerance was set to $0.02 \mathrm{Da}$. The carbamidomethylation of cysteine was set as a fixed modification, and the oxidation of methionine was considered a variable modification. Two missed trypsin cleavage sites were allowed, and the specificity of trypsin digestion was set for cleavage after lysine or arginine. Dat files $(n=54)$ were exported from Mascot software and then processed using Scaffold software (version 4.7.5, Proteome Software Inc., Portland, OR). The parameters were set as follows: both peptide and protein identifications were accepted at a false discovery rate (FDR) of less than $1.0 \%$ and proteins were identified with at least two unique peptides. Different samples were compared after normalization with the total spectra. Protein abundances at different time points were compared with spectral counting, according to previously described procedures(Old et al. 2005; Schmidt et al. 2014).

\section{Statistical analysis}

Average normalized spectral counts of each sample were used for the following statistical analysis. The levels of proteins identified on days 13 and 21 were compared with their levels on day 0 . Differential proteins were selected with the following criteria: unique peptides $\geq 2$; fold change $\geq 1.5$ or $\leq 0.67$; average spectral count in the high-abundance group $\geq 3$; comparison between two groups were conducted using two-sided, unpaired t-test; and $P$-values of group differences were adjusted by the Benjamini and Hochberg method(Benjamini \& Hochberg 1995). Group differences resulting in adjusted $P$-values $<$ 0.05 were considered statistically significant. All results are expressed as the mean \pm standard deviation.

\section{Functional enrichment analysis}

Differential proteins on days 13 and 21 were analyzed by Gene Ontology (GO) based on the biological process, cellular component and molecular function categories using the Database for Annotation, Visualization and Integrated Discovery (DAVID)(Huang da et al. 2009). The biological pathway enrichment at the two time points was analyzed with IPA software (Ingenuity Systems, Mountain View, CA, USA).

\section{Results}

\section{Characterization of rats subcutaneously inoculated with tumor cells}

A total of 12 male Wistar rats $(150 \pm 20 \mathrm{~g})$ were randomly divided into the following two groups: a control group $(n=3)$ and a group of rats subcutaneously inoculated with W256 tumor cells $(n=9)$. The body weight of these 12 rats was recorded every 3-5 days, and the daily behavior changes of the two groups were observed. The body weight of the group of rats subcutaneously inoculated with W256 tumor cells was slightly lower than that of the rats in the control group, but there were no significant differences until day 41 (Figure 2). In addition, we did not observe any detectable tumor mass in the whole period. The rats in the control group performed normal daily activities and had shiny hair. There were no significant differences in daily behavior between these two groups. 
210 Twenty-seven urine samples at three time points (days 0,13 , and 21) were used for

211 label-free LC-MS/MS quantitation. A total of 824 urinary proteins with at least 2 unique

212 peptides were identified with $<1 \%$ FDR at the protein level (Table S1). A hierarchical

213 clustering was performed by using the complete linkage method. As shown in Figure 3A, all

214 technical replications within one sample were clustered together, indicating that the technical

215 variation was smaller than the interindividual variation. In addition, all 824 proteins were

216 clustered into three clusters, which almost corresponded to the urine proteome samples from

217 the same group on day 0, day 13 and day 21 (except rat4-D0 and rat6-D21), indicating that

218 intragroup technical variation was smaller than the intergroup biological variation and

219 showing good consistency among these nine rats when inoculated with the same limited

220 number of tumor cells. Using screening criteria, 34 and 59 differential proteins were

221 identified on days 13 and 21, respectively. The overlap of these differential proteins is shown

222 by a Venn diagram in Figure 3B. Details are presented in Table 1.

\section{Functional analysis}

Functional enrichment analysis of differential proteins was performed by DAVID(Huang da et al. 2009). Differential proteins were classified into biological processes, cellular components and molecular functions. The major biological pathways of differential proteins were enriched by IPA software. A significance threshold of $P<0.05$ was used in all these representative lists.

Lists of fourteen representative biological processes on days 13 and 21 are presented in Figure 4A. Cell adhesion, negative regulation of endopeptidase activity and organ regeneration were overrepresented both on days 13 and 21. Blood coagulation, acute-phase response, autophagic cell death, positive regulation of cell proliferation, extracellular matrix organization, and response to glucose were independently overrepresented on day 13. On day 21, heterophilic cell-cell adhesion via plasma membrane cell adhesion molecules, proteolysis, positive regulation of phagocytosis and angiogenesis were independently enriched.

To identify the biological pathways involved with the differential urine proteins, IPA software was used for canonical pathway enrichment analysis. A total of 18 and 13 significant pathways were enriched on days 13 and 21, respectively (Figure 4B). Among these pathways, enriched intrinsic prothrombin activation pathway, coagulation system, acute phase response signaling, extrinsic prothrombin activation pathway and NAD phosphorylation and dephosphorylation were overrepresented on days 13 and 21. In addition, some representative pathways, such as autophagy, phagosome maturation and the role of tissue factor in cancer, were independently enriched on day 13 , and the complement system was enriched only on day 21.

The enriched cellular components and molecular functions are presented in Figure S1. 
To confirm that these differential proteins on days 13 and 21 were indeed due to the ten subcutaneously inoculated W256 tumor cells, we randomly allocated the data of these 27 samples (Number 1 to Number 27) into three groups. We tried three random allocations, and the numbers in these three groups are shown in Table 2. In each iteration, we used the data of group 1 as the control group. When we used the previous criteria to screen differential urinary proteins, it was found that the adjusted $P$-values value on days 13 and 21 were all $>0.05$. No differential proteins were selected in these three randomly allocated trials. Details are shown in Tables S2, S3 and S4.

\section{Discussion}

Urine is an early and sensitive biomarker source that has been used for the early detection of cancer either in both animal models or clinical patients(Beretov et al. 2015; Wu et al. 2017a). However, no tumor starts from thousands of tumor cells. In this study, we subcutaneously inoculated approximately ten tumor cells into each of nine rats. Unsupervised clustering analysis showed the good consistency after inoculation. A total of 34 and 59 differential proteins identified on days 13 and 21, respectively, and no urinary proteins changed after random allocation analysis.

After the functional enrichment analysis, we found that some enriched biological processes were reported to be associated with tumor progression. For example, i) cell adhesion was usually reported to show a reduced number of tumor cells since 1962(Holmberg $1962)$; ii) autophagic cell death occurs via the activation of autophagy, which has been reported to play roles in tumor suppression (Mathew et al. 2009); iii) the positive regulation of cell proliferation is a common characteristic of cancer, and the inhibition of cancer cell proliferation may serve as a potential target for cancer treatment(He et al. 2018); iv) changes in extracellular matrix organization were reported with crucial roles in cancer metastasis(Goreczny et al. 2017; Sada et al. 2016); v) positive regulation of blood coagulation was frequently reported in cancer progression(Tikhomirova et al. 2016); and vi) angiogenesis is still considered a common characteristic of tumorigenesis in many studies (Baltrunaite et al. 2017; Protopsaltis et al. 2019; Ziegler et al. 2016).

In addition, we found that some pathways were reported to play important roles in cancer. For example, i) autophagy was reported to inhibit tumor progression(Peng et al. 2016); ii) the MSP-RON signaling pathway was reported to play important roles in epithelial tumorigenesis(Ma et al. 2010) and will facilitate metastasis in prostate cancer cells(Yin et al. 2017 ) ; iii) tissue factor (TF) expressed by tumor cells was reported to facilitate lung tumor progression(Han et al. 2017); iv) upregulation of the pentose phosphate pathway (PPP) has been reported in several types of cancer(Rao et al. 2015); and v) the enriched complement system pathway was reported to enhance the metastatic process of ovarian cancer cells (Cho et al. 2016). Our results indicated that even when limited tumor cells are present in the body, the urine proteome can reflect changes associated with cancer.

When comparing differential proteins identified in our research to W256 subcutaneously tumor-bearing model(Wu et al. 2017a)and intracerebral W256 tumor model(Zhang et al. 2019), we found that the proportion of overlapping proteins was small, and more than half of the differential proteins in each of the three tumor models were unique (Figure S2). 


\section{Manuscript to be reviewed}

296 Comparing the differential proteins of the model inoculated with ten W256 cells and the

297 W256 subcutaneously tumor-bearing model showed 25 overlapping proteins. We

298 hypothesized that these small overlapping proteins may be due to the very different numbers

299 of W256 tumor cells in these two animal models. Upon comparing the differences in the urine

300 proteome between the ten tumor cell inoculated model and the intracerebral W256 tumor

301 model, only 16 differential proteins overlapped, indicating that differential proteins were very

302 different when the same tumor cells existed in different body parts. We also suppose that

303 these proteomic profiles were different because the changes observed in this study were

304 related to the very early phase of the tumor. Despite the small proportion of overlapping

305 proteins, we found that cell adhesion was enriched in GO biological process analysis using

306 either the 25 or 16 common differential proteins. This reduction in cell adhesion is a common

307 characteristic of tumor cells(Cavallaro \& Christofori 2001). These results suggest that

308 although the tumor cell number and location differ, using limited tumor cells has the potential

309 to simulate the early phase of tumor development.

$310 \quad$ Notably, it was difficult to ensure that exactly ten tumor cells were subcutaneously

311 inoculated into each of nine rats. Given the limited number of animals in this preliminary

312 study, a larger number of animals should be considered in future studies.

313

314

315

316

317

318

319

320

321

322

323

324

325

326

\section{References}

Baltrunaite K, Craig MP, Palencia Desai S, Chaturvedi P, Pandey RN, Hegde RS, and Sumanas S. 2017. ETS transcription factors Etv2 and Fli1b are required for tumor angiogenesis. Angiogenesis 20:307-323. 10.1007/s10456-017-9539-8

\section{Conclusions}

In this study, we aimed to observe changes in the urine proteome when inoculating approximately ten tumor cells into nine rats. Our results indicated that 1) the urine proteome reflects changes associated with cancer, even with a limited number of tumor cells in the body, and 2) the urine proteome reflects pathophysiological changes in the body with extremely high sensitivity, providing the potential for a very early screening process in clinical patients.

\section{Acknowledgments}

JW and YG designed the experiment; JW performed the experiments, performed the LC-MS/MS analysis, analyzed the data and wrote the manuscript; WM performed the animal experiments. All authors read and approved the final manuscript.

Benjamini Y, and Hochberg Y. 1995. Controlling the false discovery rate: a practical and powerful approach to multiple testing. Journal of the Royal Statistical Society Series B 57:289-300.

Beretov J, Wasinger VC, Millar EK, Schwartz P, Graham PH, and Li Y. 2015. Proteomic Analysis of Urine to Identify Breast Cancer Biomarker Candidates Using a Label-Free LC-MS/MS Approach. PLoS One 10:e0141876. 10.1371/journal.pone.0141876

Cavallaro U, and Christofori G. 2001. Cell adhesion in tumor invasion and metastasis: loss of the glue is not enough. Biochimica Et Biophysica Acta 1552:39-45. 
Cho MS, Rupaimoole R, Choi HJ, Noh K, Chen J, Hu Q, Sood AK, and
Afshar-Kharghan V. 2016. Complement Component 3 Is Regulated by TWIST1 and Mediates Epithelial-Mesenchymal Transition. Journal of Immunology 196:1412-1418. 10.4049/jimmunol.1501886

Ferreira R, Oliveira P, Martins T, Magalhaes S, Trindade F, Pires MJ, Colaco B, Barros A, Santos L, Amado F, and Vitorino R. 2015. Comparative proteomic analyses of urine from rat urothelial carcinoma chemically induced by exposure to N-butyl-N-(4-hydroxybutyl)-nitrosamine. Molecular bioSystems 11:1594-1602. $10.1039 / \mathrm{c} 4 \mathrm{mb} 00606 \mathrm{~b}$

Gao Y. 2013. Urine-an untapped goldmine for biomarker discovery? Science China Life Science 56:1145-1146. 10.1007/s11427-013-4574-1

Gao Y. 2014. Roadmap to the Urine Biomarker Era. MOJ Proteomics Bioinformatics 1:00005.

Goreczny GJ, Ouderkirk-Pecone JL, Olson EC, Krendel M, and Turner CE. 2017. Hic-5 remodeling of the stromal matrix promotes breast tumor progression. Oncogene 36:2693-2703. 10.1038/onc.2016.422

Han X, Zha H, Yang F, Guo B, and Zhu B. 2017. Tumor-Derived Tissue Factor Aberrantly Activates Complement and Facilitates Lung Tumor Progression via Recruitment of Myeloid-Derived Suppressor Cells. International Journal of Molecular Science 18: pii:E22. 10.3390/ijms18010022

He J, Chen Y, Cai L, Li Z, and Guo X. 2018. UBAP2L silencing inhibits cell proliferation and G2/M phase transition in breast cancer. Breast Cancer 25:224-232. $10.1007 / \mathrm{s} 12282-017-0820-\mathrm{x}$

Holmberg B. 1962. Inhibition of cellular adhesion and pseudopodia formation by a dialysable factor from tumour fluids. Nature 195:45-47. 10.1038/195045a0

Huang da W, Sherman BT, and Lempicki RA. 2009. Systematic and integrative analysis of large gene lists using DAVID bioinformatics resources. Nature Protocols 4:44-57. 10.1038/nprot.2008.211

Li M, Zhao M, and Gao Y. 2014. Changes of proteins induced by anticoagulants can be more sensitively detected in urine than in plasma. Science China Life Science 57:649-656. 10.1007/s11427-014-4661-y

Ma Q, Zhang K, Yao HP, Zhou YQ, Padhye S, and Wang MH. 2010. Inhibition of MSP-RON signaling pathway in cancer cells by a novel soluble form of RON comprising the entire sema sequence. International Journal of Oncology 36:1551-1561. 10.3892/ijo_00000642

Mathew R, Karp CM, Beaudoin B, Vuong N, Chen G, Chen HY, Bray K, Reddy A, Bhanot G, Gelinas C, Dipaola RS, Karantza-Wadsworth V, and White E. 2009. Autophagy suppresses tumorigenesis through elimination of p62. Cell 137:1062-1075. 10.1016/j.cell.2009.03.048

Ni Y, Zhang F, An M, Yin W, and Gao Y. 2018a. Early candidate biomarkers found from urine of glioblastoma multiforme rat before changes in MRI. Science China Life Science 61:982-987. 10.1007/s11427-017-9201-0. 


\section{Manuscript to be reviewed}

382

383

384

385

386

387

388

389

390

391

392

393

394

395

396

397

398

399

400

401

402

403

404

405

406

407

408

409

410

411

412

413

414

415

416

417

418

419

420

421

422

423

424

Resing KA, and Ahn NG. 2005. Comparison of label-free methods for quantifying human proteins by shotgun proteomics. Molecularl \&Cellular Proteomics 4:1487-1502. 10.1074/mcp.M500084-MCP200

Peng Y, Miao H, Wu S, Yang W, Zhang Y, Xie G, Xie X, Li J, Shi C, Ye L, Sun W, Wang L, Liang H, and Ou J. 2016. ABHD5 interacts with BECN1 to regulate autophagy and tumorigenesis of colon cancer independent of PNPLA2. Autophagy 12:2167-2182. $10.1080 / 15548627.2016 .1217380$

Protopsaltis NJ, Liang W, Nudleman E, and Ferrara N. 2019. Interleukin-22 promotes tumor angiogenesis. Angiogenesis 22:311-323. 10.1007/s10456-018-9658-x

Rao X, Duan X, Mao W, Li X, Li Z, Li Q, Zheng Z, Xu H, Chen M, Wang PG, Wang Y, Shen B, and Yi W. 2015. O-GlcNAcylation of G6PD promotes the pentose phosphate pathway and tumor growth. Nature Communications 6:8468. 10.1038/ncomms9468

Sada M, Ohuchida K, Horioka K, Okumura T, Moriyama T, Miyasaka Y, Ohtsuka T, Mizumoto K, Oda Y, and Nakamura M. 2016. Hypoxic stellate cells of pancreatic cancer stroma regulate extracellular matrix fiber organization and cancer cell motility. Cancer Letters 372:210-218. 10.1016/j.canlet.2016.01.016

Schmidt C, Gronborg M, Deckert J, Bessonov S, Conrad T, Luhrmann R, and Urlaub H. 2014. Mass spectrometry-based relative quantification of proteins in precatalytic and catalytically active spliceosomes by metabolic labeling (SILAC), chemical labeling (iTRAQ), and label-free spectral count. RNA 20:406-420. 10.1261/rna.041244.113

Tikhomirova I, Petrochenko E, Malysheva Y, Ryabov M, and Kislov N. 2016. Interrelation of blood coagulation and hemorheology in cancer. Clinical Hemorheology and Microcirculation 64:635-644. 10.3233/ch-168037

Wisniewski JR, Zougman A, Nagaraj N, and Mann M. 2009. Universal sample preparation method for proteome analysis. Nature Methods 6:359-362. 10.1038/nmeth.1322

Wu J, and Gao Y. 2015. Physiological conditions can be reflected in human urine proteome and metabolome. Expert Review of Proteomics 12:623-636. $10.1586 / 14789450.2015 .1094380$

Wu J, Guo Z, and Gao Y. 2017a. Dynamic changes of urine proteome in a Walker 256 tumor-bearing rat model. Cancer Medicine 6:2713-2722. 10.1002/cam4.1225

Wu J, Li X, Zhao M, Huang H, Sun W, and Gao Y. 2017b. Early Detection of Urinary Proteome Biomarkers for Effective Early Treatment of Pulmonary Fibrosis in a Rat Model. Proteomics Clinical Applications 11: 11-12.10.1002/prca.201700103

Yin B, Liu Z, Wang Y, Wang X, Liu W, Yu P, Duan X, Liu C, Chen Y, Zhang Y, Pan X, Yao H, Liao Z, and Tao Z. 2017. RON and c-Met facilitate metastasis through the ERK signaling pathway in prostate cancer cells. Oncology Reports 37:3209-3218. 10.3892/or.2017.5585

Zhang F, Ni Y, Yuan Y, Yin W, and Gao Y. 2018a. Early urinary candidate biomarker discovery in a rat thioacetamide-induced liver fibrosis model. Science China Life Science 61:1369-1381. 10.1007/s11427-017-9268-y

Zhang F, Wei J, Li X, Ma C, and Gao Y. 2018b. Early Candidate Urine Biomarkers for Detecting Alzheimer's Disease Before Amyloid-beta Plaque Deposition in an APP (swe)/PSEN1dE9 Transgenic Mouse Model. Journal of Alzheimer's Disease Peer) reviewing PDF | (2019:05:37656:1:1:CHECK 31 Jul 2019) 
66:613-637. 10.3233/jad-180412

Zhang H, Cao J, Li L, Liu Y, Zhao H, Li N, Li B, Zhang A, Huang H, Chen S, Dong M, Yu L, Zhang J, and Chen L. 2015. Identification of urine protein biomarkers with the potential for early detection of lung cancer. Scientific Reports 5:11805. 10.1038/srep11805

Zhang L, Li Y, and Gao Y. 2018c. Early changes in the urine proteome in a diethyldithiocarbamate-induced chronic pancreatitis rat model. Journal of Proteomics 186:8-14. 10.1016/j.jprot.2018.07.015

Zhang L, Li Y, Meng W, Ni Y, and Gao Y. 2019. Dynamic urinary proteomic analysis in a Walker 256 intracerebral tumor model. Cancer Medicine 8:3553-3565. $10.1002 /$ cam 4.2240

Zhao M, Wu J, Li X, and Gao Y. 2018. Urinary candidate biomarkers in an experimental autoimmune myocarditis rat model. Journal of Proteomics 179:71-79. 10.1016/j.jprot.2018.02.032

Ziegler ME, Hatch MM, Wu N, Muawad SA, and Hughes CC. 2016. mTORC2 mediates CXCL12-induced angiogenesis. Angiogenesis 19:359-371. 


\section{Manuscript to be reviewed}

468 Figure legends

469

470

471

472

473

474

475

476

477

478

479

480

481

482

483

484

485

486

487

488

489

490

491

492

493

494

495

496

Figure 1. Workflow of protein identification in rats subcutaneously inoculated with ten tumor cells. Urine was collected on days 0,13 and 21 after inoculation with tumor cells. Urinary proteins were extracted, digested, and identified by liquid chromatography coupled with tandem mass spectrometry (LC-MS/MS). Functional enrichment analysis of differential proteins was performed by DAVID and IPA.

Figure 2. Body weight changes between the rats subcutaneously inoculated with tumor cells and the control rats.

Figure 3. Proteomic analysis of urine samples on days 13 and 21 in rats subcutaneously inoculated with tumor cells. (A) Unsupervised cluster analysis of all proteins identified by LC-MS/MS. (B) Overlap evaluation of the differential proteins identified on days 13 and 21.

Figure 4. Functional analysis of differential proteins on days 13 and 21. (A) Dynamic changes in biological processes on day 13. (B) Dynamic changes in biological processes on day 21. (C) Dynamic changes in pathways on day 13. (D) Dynamic changes in pathways on day 21 .

Table 1. Differential proteins identified on day 13 and day 21.

Table 2. Random allocation of the twenty-seven urine samples.

Peer) reviewing PDF | (2019:05:37656:1:1:CHECK 31 Jul 2019) 


\section{Figure 1}

Workflow of protein identification in ten tumor cells subcutaneously inoculated in rats.

Urine was collected on days 0,13 and 21 after inoculation with tumor cells. Urinary proteins were extracted, digested, and identified by liquid chromatography coupled with tandem mass spectrometry (LC-MS/MS). Functional enrichment analysis of differential proteins was performed by DAVID and IPA. 
W256 tumor cells $(\mathrm{n} \approx 10)$

$\downarrow$

Day 0 Day 13 Day 21

9 Rats $\times 3$ time points

\section{Urinary protein extraction}

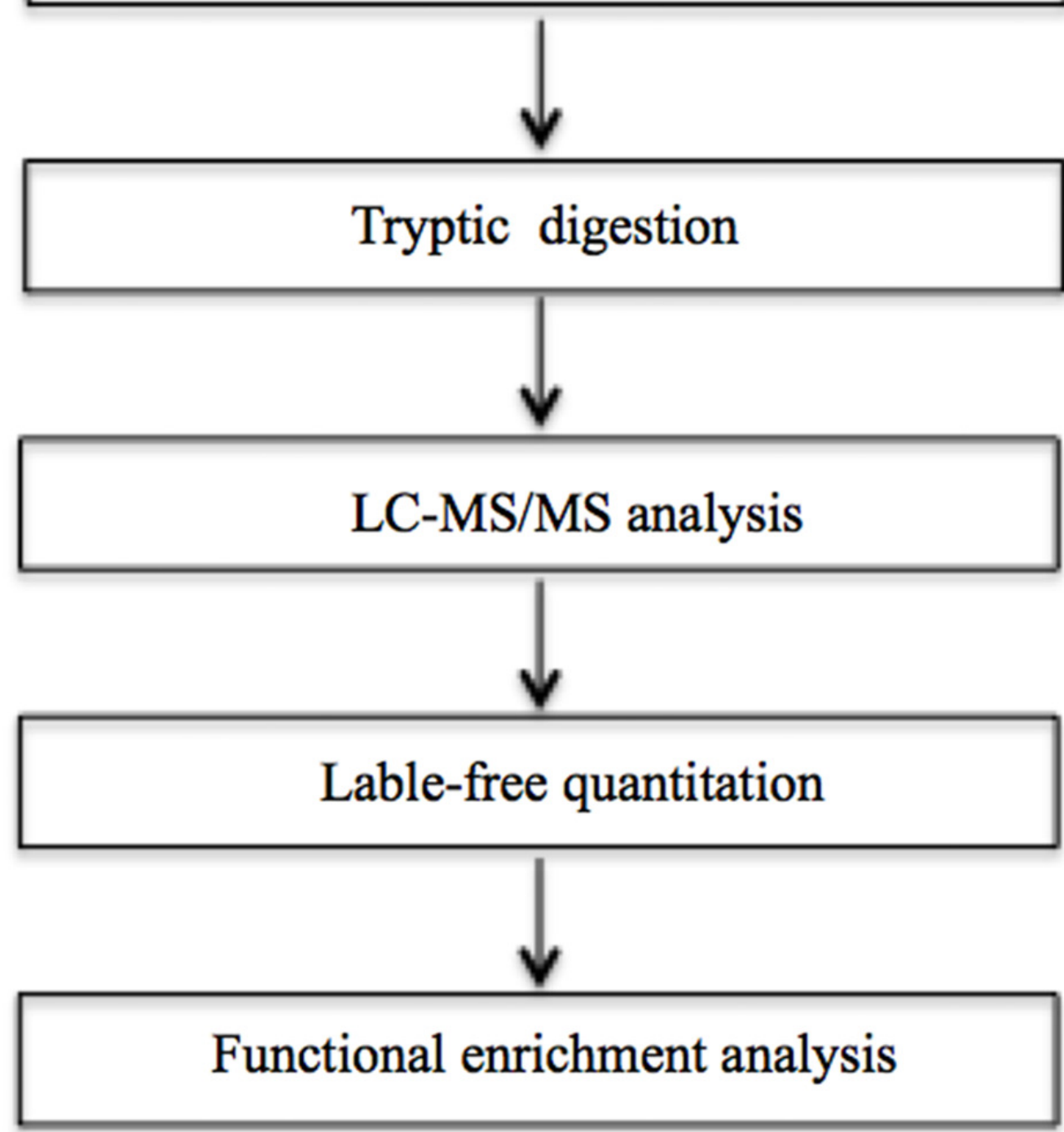


Figure 2

Body weight changes between the rats subcutaneously inoculated with tumor cells and the control rats.

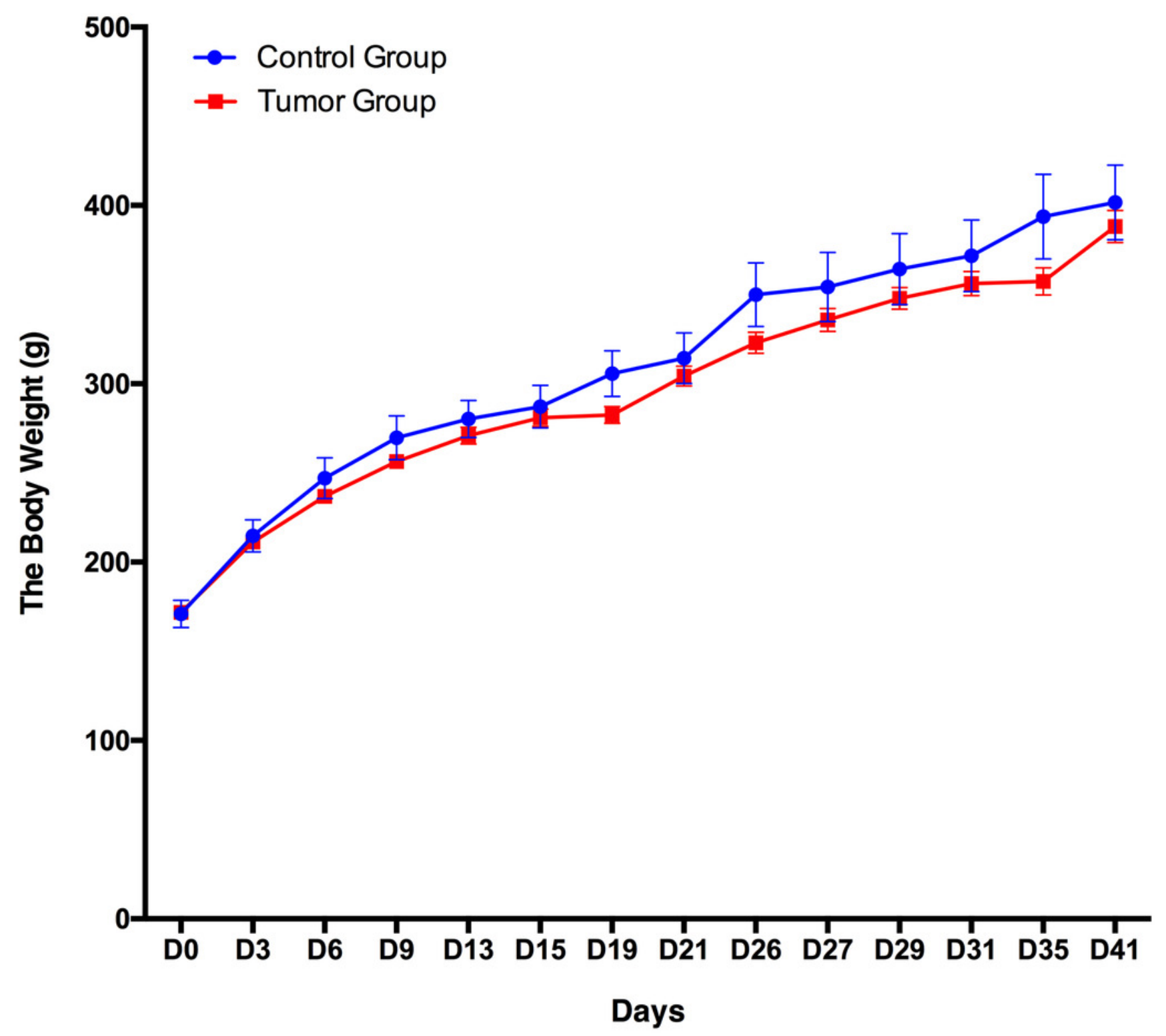


Figure 3

Proteomic analysis of urine samples on days 13 and 21 in rats subcutaneously inoculated with tumor cells.

(A) Unsupervised cluster analysis of all proteins identified by LC-MS/MS. (B) Overlap evaluation of the differential proteins identified on days 13 and 21. 
A

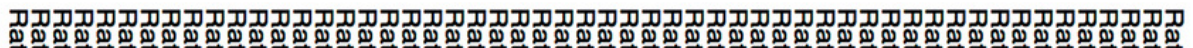

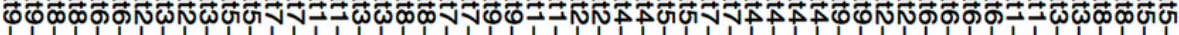

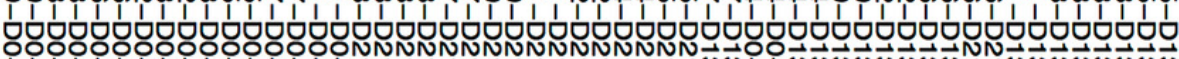
$\begin{array}{lll}1 & 1 \\ 1 & 1\end{array}$

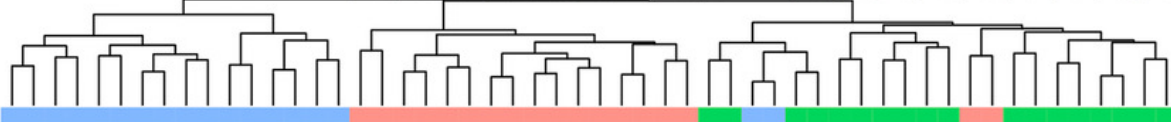
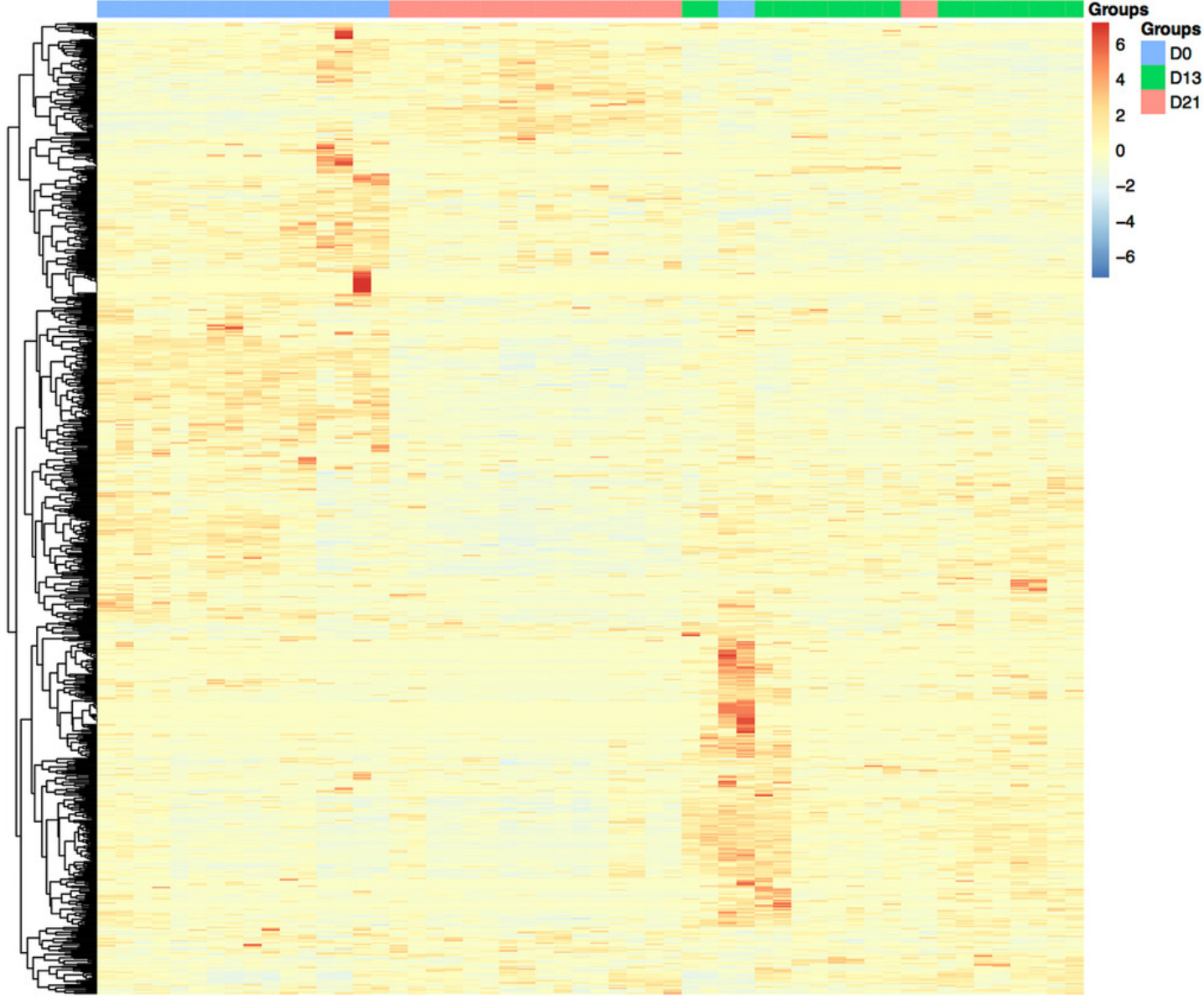

B

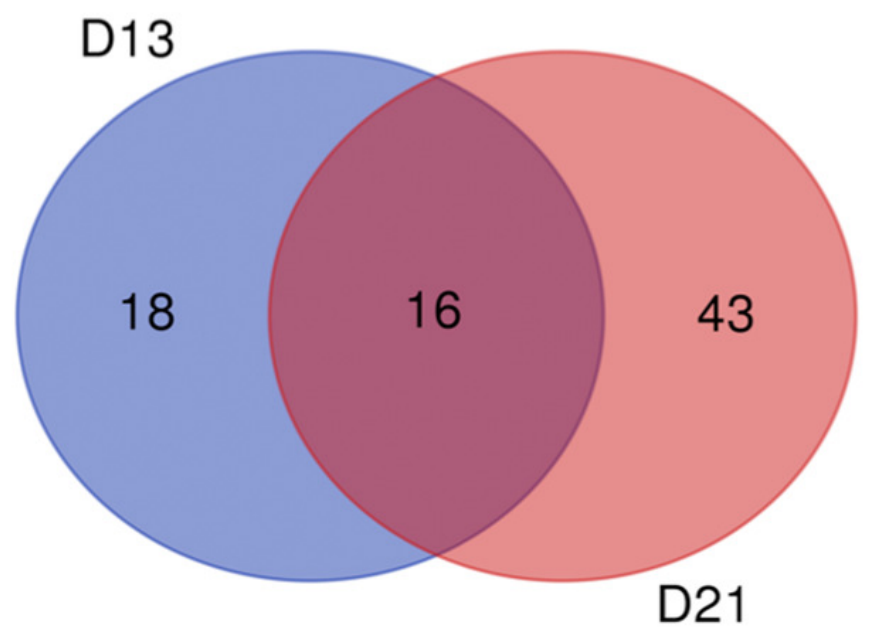




\section{Figure 4}

\section{Functional analysis of differential proteins on days 13 and 21.}

\section{(A) Dynamic changes in biological processes on day 13. (B) Dynamic changes in biological}

processes on day 21. (C) Dynamic changes in pathways on day 13. (D) Dynamic changes in pathways on day 21 .

\section{Biological Process}

A

\section{D13}

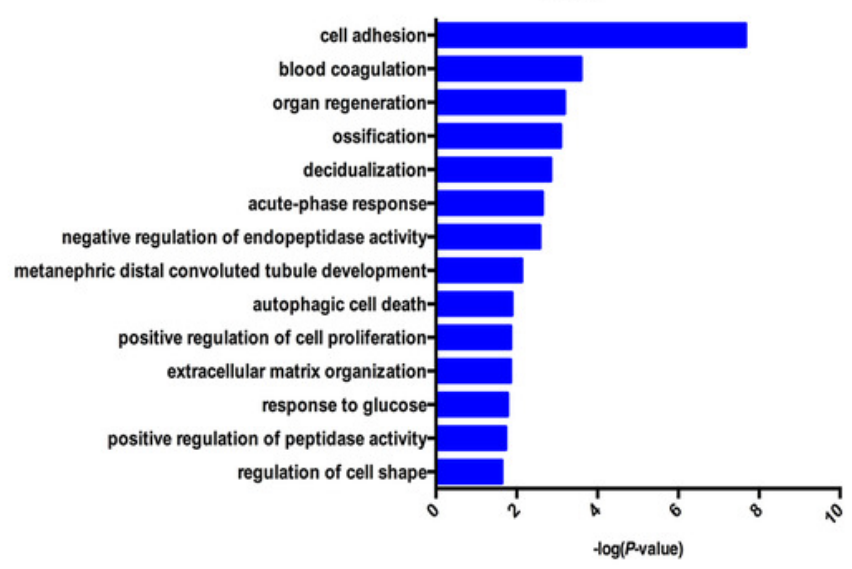

Pathways

C

D13

Acute Phase Response Signaling-

Extrinsic Prothrombin Activation PathwayLXR/RXR ActivationFXR/RXR ActivationCoagulation SystemIntrinsic Prothrombin Activation PathwayHepatic Fibrosis / Hepatic Stellate Cell Activationautophagy-

Thyroid Hormone BiosynthesisMSP-RON Signaling PathwayRole of Macrophages, Fibroblasts and Endothelial Cells in Rheumatoid Arthritis Hematopoiesis from Multipotent Stem CellsNAD Phosphorylation and DephosphorylationRole of Tissue Factor in CancerPhagosome MaturationAryl Hydrocarbon Receptor SignalingMethylglyoxal Degradation IIINAD Salvage Pathway II

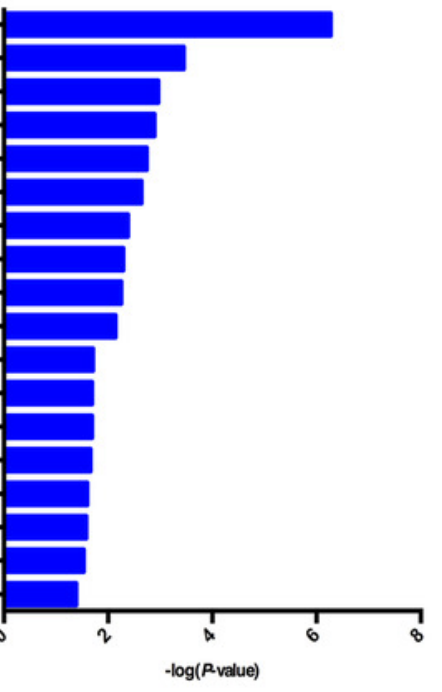

B

D21

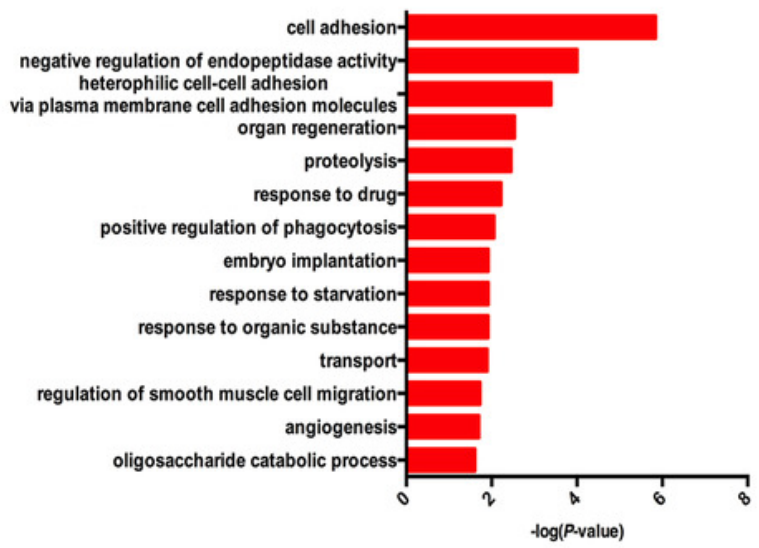




\section{Table $\mathbf{1}$ (on next page)}

Differential proteins identified on day 13 and day 21 . 
Table 1. Differentially proteins identified on day 13 and day 21.

\begin{tabular}{|c|c|c|c|c|c|c|}
\hline \multirow{2}{*}{$\begin{array}{l}\text { UniProt } \\
\text { ID }\end{array}$} & \multirow{2}{*}{$\begin{array}{l}\text { Human } \\
\text { ortholog }\end{array}$} & \multirow[t]{2}{*}{ Description } & \multirow[t]{2}{*}{ Trends } & \multirow{2}{*}{$\begin{array}{c}\text { ANOVA } \\
P \text {-value }\end{array}$} & \multicolumn{2}{|c|}{$\begin{array}{c}\text { Average fold } \\
\text { change }\end{array}$} \\
\hline & & & & & Day 13 & Day 21 \\
\hline P97603 & Q92859 & Neogenin (fragment) & $\downarrow$ & $<0.00010$ & 0.43 & 0.21 \\
\hline O70535 & P42702 & $\begin{array}{l}\text { Leukemia inhibitory factor } \\
\text { receptor }\end{array}$ & $\downarrow$ & $<0.00010$ & 0.29 & 0.35 \\
\hline Q9ESS6 & P50895 & Basal cell adhesion molecule & $\downarrow$ & $<0.00010$ & 0.56 & 0.40 \\
\hline P02761 & NO & $\begin{array}{l}\text { Cluster of Major urinary } \\
\text { protein }\end{array}$ & $\uparrow$ & $<0.00010$ & 4.70 & 7.59 \\
\hline P20611 & P11117 & Lysosomal acid phosphatase & $\downarrow$ & $<0.00010$ & 0.56 & 0.46 \\
\hline Q9JI85 & P80303 & Nucleobindin-2 & $\uparrow$ & 0.00018 & 2.74 & 3.69 \\
\hline P07897 & P16112 & Aggrecan core protein & $\downarrow$ & $<0.00010$ & 0.23 & 0.30 \\
\hline Q63416 & Q06033 & $\begin{array}{c}\text { Inter-alpha-trypsin inhibitor } \\
\text { heavy chain } \mathrm{H} 3\end{array}$ & $\downarrow$ & $<0.00010$ & 0.32 & 0.23 \\
\hline P06760 & P08236 & Beta-glucuronidase & $\uparrow$ & 0.0073 & 3.56 & 4.55 \\
\hline $\mathrm{O} 35112$ & Q13740 & CD166 antigen & $\downarrow$ & $<0.00010$ & 0.41 & 0.33 \\
\hline Q63556 & NO & $\begin{array}{l}\text { Serine protease inhibitor } \\
\qquad \text { A3M (fragment) }\end{array}$ & $\downarrow$ & $<0.00010$ & 0.47 & 0.48 \\
\hline P26453 & P35613 & Basigin & $\downarrow$ & 0.00094 & 0.23 & 0.21 \\
\hline P27590 & P07911 & Uromodulin & $\uparrow$ & $<0.00010$ & 2.31 & 2.65 \\
\hline P35444 & P49747 & $\begin{array}{l}\text { Cartilage oligomeric matrix } \\
\text { protein }\end{array}$ & $\downarrow$ & $<0.00010$ & 0.27 & 0.42 \\
\hline Q9EPF2 & P43121 & $\begin{array}{l}\text { Cell surface glycoprotein } \\
\text { MUC18 }\end{array}$ & $\downarrow$ & $<0.00010$ & 0.18 & 0.08 \\
\hline P13596 & P13591 & $\begin{array}{c}\text { Neural cell adhesion } \\
\text { molecule } 1\end{array}$ & $\downarrow$ & $<0.00010$ & 0.28 & 0.36 \\
\hline P18292 & P00734 & Prothrombin & $\downarrow$ & $<0.00010$ & 0.60 & - \\
\hline P07154 & P07711 & Cathepsin L1 & $\downarrow$ & 0.00021 & 0.40 & - \\
\hline Q63467 & P04155 & Trefoil factor 1 & $\downarrow$ & 0.00026 & 0.50 & - \\
\hline D3ZTE0 & P00748 & Coagulation factor XII & $\downarrow$ & $<0.00010$ & 0.25 & - \\
\hline Q5HZW5 & Q9NPF0 & CD320 antigen & $\downarrow$ & $<0.00010$ & 0.45 & - \\
\hline Q8JZQ0 & Р09603 & $\begin{array}{l}\text { Macrophage colony- } \\
\text { stimulating factor } 1\end{array}$ & $\downarrow$ & 0.00013 & 0.42 & - \\
\hline P26644 & P02749 & Beta-2-glycoprotein 1 & $\downarrow$ & 0.00016 & 0.46 & - \\
\hline P24268 & P07339 & Cathepsin D & $\uparrow$ & $<0.00010$ & 1.56 & - \\
\hline P04937 & $\mathrm{P} 02751$ & Fibronectin & $\downarrow$ & $<0.00010$ & 0.61 & - \\
\hline Q63530 & Q96BW5 & $\begin{array}{c}\text { Phosphotriesterase-related } \\
\text { protein }\end{array}$ & $\uparrow$ & 0.0004 & 3.18 & - \\
\hline
\end{tabular}




\begin{tabular}{|c|c|c|c|c|c|c|}
\hline P97546 & Q9Y639 & Neuroplastin & $\downarrow$ & $<0.00010$ & 0.47 & - \\
\hline P07171 & P05937 & Cluster of Calbindin & $\downarrow$ & $<0.00010$ & 0.05 & - \\
\hline P24090 & P02765 & Alpha-2-HS-glycoprotein & $\downarrow$ & 0.00026 & 0.47 & - \\
\hline P38918 & O95154 & $\begin{array}{l}\text { Aflatoxin B1 aldehyde } \\
\text { reductase member } 3\end{array}$ & $\uparrow$ & 0.0028 & 3.36 & - \\
\hline P08592 & NO & Amyloid beta A4 protein & $\downarrow$ & $<0.00010$ & 0.56 & - \\
\hline Q62930 & P02748 & Complement component C9 & $\downarrow$ & 0.0055 & 0.49 & - \\
\hline Q91XN4 & Q13145 & $\begin{array}{l}\text { BMP and activin membrane- } \\
\text { bound inhibitor homolog }\end{array}$ & $\downarrow$ & 0.003 & 0.55 & - \\
\hline Q9JLS4 & Q6FHJ7 & $\begin{array}{l}\text { Secreted frizzled-related } \\
\text { protein } 4\end{array}$ & $\downarrow$ & 0.00053 & 0.52 & - \\
\hline Q5I0D5 & Q9H008 & $\begin{array}{l}\text { Phospholysine } \\
\text { phosphohistidine inorganic } \\
\text { pyrophosphate phosphatase }\end{array}$ & $\downarrow$ & $<0.00010$ & - & 0.20 \\
\hline P80202 & P36896 & Activin receptor type-1B & $\uparrow$ & $<0.00010$ & - & 1.76 \\
\hline $\begin{array}{l}\text { Q9WUW } \\
3\end{array}$ & P05156 & Complement factor I & $\downarrow$ & $<0.00010$ & - & 0.42 \\
\hline P07151 & P61769 & Beta-2-microglobulin & $\uparrow$ & $<0.00010$ & - & 1.65 \\
\hline Q642A7 & $\begin{array}{c}\text { Q8WW5 } \\
2\end{array}$ & Protein FAM151A & $\downarrow$ & 0.00045 & - & 0.58 \\
\hline Q63678 & P25311 & Zinc-alpha-2-glycoprotein & $\downarrow$ & $<0.00010$ & - & 0.20 \\
\hline Q9EQV6 & O14773 & Tripeptidyl-peptidase 1 & $\downarrow$ & 0.0033 & - & 0.30 \\
\hline Q63751 & NO & Vomeromodulin (fragment) & $\downarrow$ & 0.012 & - & 0.31 \\
\hline P04073 & P20142 & Gastricsin & $\downarrow$ & 0.00044 & - & 0.27 \\
\hline O88917 & NO & $\begin{array}{l}\text { Adhesion } \mathrm{G} \text { protein-coupled } \\
\text { receptor L1 }\end{array}$ & $\downarrow$ & $<0.00010$ & - & 0.42 \\
\hline P36374 & NO & $\begin{array}{l}\text { Prostatic glandular } \\
\text { kallikrein-6 }\end{array}$ & $\uparrow$ & 0.0034 & - & 1.78 \\
\hline Q63475 & Q92932 & $\begin{array}{l}\text { Receptor-type tyrosine- } \\
\text { protein phosphatase N2 }\end{array}$ & $\downarrow$ & $<0.00010$ & - & 6.25 \\
\hline Q01460 & Q01459 & Di-N-acetylchitobiase & $\downarrow$ & $<0.00010$ & - & 0.61 \\
\hline Q99PW3 & Q99519 & Sialidase-1 & $\downarrow$ & 0.00025 & - & 0.18 \\
\hline P85971 & O95336 & 6-phosphogluconolactonase & $\downarrow$ & 0.0005 & - & 0.39 \\
\hline P47820 & $\mathrm{P} 12821$ & $\begin{array}{c}\text { Angiotensin-converting } \\
\text { enzyme }\end{array}$ & $\downarrow$ & 0.0016 & - & 0.13 \\
\hline Q1WIM1 & Q8NFZ8 & Cell adhesion molecule 4 & $\downarrow$ & $<0.00010$ & - & 0.24 \\
\hline P05545 & NO & $\begin{array}{l}\text { Serine protease inhibitor } \\
\qquad \mathrm{A} 3 \mathrm{~K}\end{array}$ & $\downarrow$ & $<0.00010$ & - & 0.61 \\
\hline P81828 & NO & Urinary protein 2 & $\uparrow$ & $<0.00010$ & - & 1.96 \\
\hline
\end{tabular}




\begin{tabular}{|c|c|c|c|c|c|c|}
\hline P31211 & P08185 & $\begin{array}{l}\text { Corticosteroid-binding } \\
\text { globulin }\end{array}$ & $\downarrow$ & 0.00011 & - & 0.48 \\
\hline P04785 & P07237 & Protein disulfide-isomerase & $\downarrow$ & $<0.00010$ & - & 0.17 \\
\hline P06866 & P00739 & Haptoglobin & $\uparrow$ & 0.00036 & - & 1.68 \\
\hline Q562C9 & Q9BV57 & $\begin{array}{c}\text { 1,2-dihydroxy-3-keto-5- } \\
\text { methylthiopentene } \\
\text { dioxygenase }\end{array}$ & $\downarrow$ & 0.0057 & - & 0.43 \\
\hline Q4V885 & Q5KU26 & Collectin-12 & $\uparrow$ & 0.0011 & - & 2.28 \\
\hline P21704 & P24855 & Deoxyribonuclease-1 & $\downarrow$ & $<0.00010$ & - & 0.56 \\
\hline P63018 & P11142 & $\begin{array}{l}\text { Cluster of heat shock } \\
\text { cognate } 71 \mathrm{kDa} \text { protein }\end{array}$ & $\downarrow$ & 0.00045 & - & 0.56 \\
\hline P00714 & P00709 & Alpha-lactalbumin & $\uparrow$ & $<0.00010$ & - & 3.77 \\
\hline Q6AYE5 & Q86UD1 & Out at first protein homolog & $\downarrow$ & $<0.00010$ & - & 0.16 \\
\hline Q68FQ2 & Q9BX67 & $\begin{array}{c}\text { Junctional adhesion } \\
\text { molecule } \mathrm{C}\end{array}$ & $\downarrow$ & 0.0011 & - & 0.53 \\
\hline O55004 & P34096 & Ribonuclease 4 & $\uparrow$ & 0.00023 & - & 2.13 \\
\hline Q641Z7 & Q92484 & $\begin{array}{c}\text { Acid sphingomyelinase-like } \\
\text { phosphodiesterase } 3 \mathrm{a}\end{array}$ & $\downarrow$ & $<0.00010$ & - & 0.18 \\
\hline O70244 & O60494 & Cubilin & $\downarrow$ & $<0.00010$ & - & 0.25 \\
\hline Q00657 & Q6UVK1 & $\begin{array}{c}\text { Chondroitin sulfate } \\
\text { proteoglycan } 4\end{array}$ & $\downarrow$ & $<0.00010$ & - & 0.28 \\
\hline P29598 & P00749 & $\begin{array}{c}\text { Urokinase-type plasminogen } \\
\text { activator }\end{array}$ & $\downarrow$ & $<0.00010$ & - & 0.62 \\
\hline P01026 & P01024 & Complement $\mathrm{C} 3$ & $\downarrow$ & 0.0071 & - & 0.42 \\
\hline P53813 & P07225 & $\begin{array}{l}\text { Vitamin K-dependent } \\
\text { protein } \mathrm{S}\end{array}$ & $\downarrow$ & 0.00096 & - & 0.29 \\
\hline Q80WD1 & Q86UN3 & Reticulon-4 receptor-like 2 & $\downarrow$ & 0.017 & - & 0.04 \\
\hline P62986 & P62987 & $\begin{array}{l}\text { Ubiquitin-60S ribosomal } \\
\text { protein } \mathrm{L} 40\end{array}$ & $\uparrow$ & 0.0011 & - & 1.81 \\
\hline Q9R0T4 & P12830 & Cadherin-1 & $\downarrow$ & $<0.00010$ & - & 0.47 \\
\hline P08937 & NO & Odorant-binding protein & $\uparrow$ & 0.002 & - & 1.51 \\
\hline P05544 & NO & $\begin{array}{l}\text { Serine protease inhibitor } \\
\text { A3L }\end{array}$ & $\downarrow$ & 0.00026 & - & 0.64 \\
\hline Q05175 & P80723 & Brain acid soluble protein 1 & $\uparrow$ & $<0.00010$ & - & 3.69 \\
\hline P61972 & P61970 & Nuclear transport factor 2 & $\downarrow$ & 0.0024 & - & 0.54 \\
\hline
\end{tabular}


Table 2 (on next page)

Random allocation of the twenty-seven urine samples. 
Table 2. Random allocation of the twenty-seven urine samples

\begin{tabular}{ccccc}
\hline $\begin{array}{c}\text { Randomly } \\
\text { allocated }\end{array}$ & Group 1 & Group 2 & Group 3 & $\begin{array}{c}\text { Adjusted P- } \\
\text { value }\end{array}$ \\
\hline \multirow{2}{*}{1} & $1,4,5,12,13$, & $2,3,7,11,15$, & $6,8,9,10,16$, & NO \\
& $14,19,20,21$ & $17,18,22,23$ & $24,25,26,27$ & \\
2 & $1,2,3,12,13$, & $4,5,6,10,11$, & $7,8,9,16,17$, & NO \\
& $15,25,26,27$ & $14,19,20,21$ & $18,22,23,24$ & NO \\
\hline \multirow{2}{*}{3} & $1,7,9,11,13$, & $3,4,5,10,17$, & $2,6,8,12,14$, & \\
\hline
\end{tabular}

2 Numbers 1-9 represent Rat 1-D0 to Rat 9-D0; Numbers 10-18 represent Rat 1-D13 to Rat 9-D13; Numbers 19-27

3 represent Rat 1-D21 to Rat 9-D21.

4 